\title{
Physicians' discontent: burnout and the deterioration of medical practice
}

\author{
Bernardo Moreno-Jiménez, ${ }^{1}$ María del Carmen Yeo Ayala, ${ }^{2}$ Ana Fresán-Orellana ${ }^{3}$
}

Departamento de Psicología Biológica y de la Salud, Universidad Autónoma de Madrid, España. 2 Centro Universitario de Ciencias de la Salud, Universidad de Guadalajara, México.

3 Subdirección de Investigaciones Clínicas, Instituto Nacional de Psiquiatría Ramón de la Fuente Muñiz.

Correspondence:

Bernardo Moreno-Jiménez

Departamento de Psicología Biológica y de la Salud, Facultad de Psicología, Universidad Autónoma de Madrid, España.

Ciudad Universitaria de Cantoblanco.

Calle Ivan Pavlov 6

28049 Madrid, España.

Phone: (+34) 91497 - 5185

Email: bernardo.moreno@uam.es

\section{Citation:}

Moreno-liménez, B., Yeo Ayala, M C., \& Fresán-Orellana, A. (2020) Physicians' discontent: burnout and the deterioration of medical practice. Salud Mental, 43(5), 191-193.

DOI: $10.17711 /$ SM.0185-3325.2020.026
It is a well-known fact among professionals that medical well-being has eroded in recent decades (Schrijver, Brady, \& Trockel, 2016), together with the mental health of medical professionals. The root of medical mental problems is clinical practice. Practicing medicine is not an easy task since it involves constantly confronting disease, pain, suffering, the progressive loss of quality of life, and even death (Boorse, 2016). Eventhough coping with and accompanying these processes to suppress or alleviate them provides satisfaction and personal fulfillment (Janus et al., 2008), it also has a cost. Being involved in improving the health of others often compromises the physical and mental health of physicians, and their professional quality of life (Mingote Adán, Moreno Jiménez, Gálvez Herrer, 2004). Engaging in a difficult clinical relationship is compounded by the problems of a system of working conditions that contribute to medical mental exhaustion (Milner et al., 2017).

Medical stress originates in a culture of medical practice learned at medical schools and in the individual psychology of the person who has chosen medicine as a career. Medical culture encourages the development of a strong ethics of responsibility and the obligation to persist in one's efforts (Myers \& Gabbard, 2008). The training that physicians receive facilitates "addictive" work patterns (Landrigan, Barger, Cade, Ayas, \& Czeisler, 2006). Moreover, the physician's psychology has been based on the development of qualities such as idealism, self-denial, a sense of responsibility, and perfectionism (Gazelle, Liebschutz, \& Riess, 2015). Although these are positive, they can lead to commitments and demands that can undermine long-term personal well-being and lead to deterioration and mental instability (Lacy \& Chan, 2018). The literature also suggests that physicians are prone to neglect self-care (Wallace, Lemaire, \& Ghali, 2009).

It is hardly surprising then that various mental health problems have been described in physicians (Myers \& Gabbard, 2008). Data indicate that female physicians are at increased risk for poor mental health outcomes (Mache, Klapp, \& Groneberg, 2014). Certain contexts and practices can prompt feelings of discouragement, irritation, and disappointment, which can lead to the loss of personal well-being and the ability to enjoy the profession itself (Miller, 2016). The most common disorders involve mood, anxiety, and substance use. Other more serious ones such as depressive disorders, ideation, and suicidal behavior are more frequent than in other professions (Center et al., 2003). More important than mental disorders themselves has been the attitude towards them. Mental problems have traditionally been viewed as a stigma that could not be disclosed or be personally accepted by the medical professional (Wallace, 2012).

The burden of work stress in medical practice is higher than that of other professions (Firth-Cozens, 2003). When medical stress becomes chronic, there is a high likelihood of it turning into burnout. As the Federation of State Medical Boards (FSMB, 2018) has declared, it is not easy to find a better concept than that of burnout to describe the widespread discontent affecting physicians, the practice of medicine, and the health system as a whole. To speak of medical burnout is to speak of chronic medical stress, but with a greater scope. Burnout expresses chronic discontent with medical practice, the health systems in which it is practiced, and the culture of practicing medicine. It involves the profound depletion of available mental energy and, in many cases, a breakdown of internal equilibrium (Tyssen, 
2018). It has been operatively presented as a response with three components: emotional exhaustion, depersonalization, and lack of personal fulfillment (Maslach \& Jackson, 1981).

Although burnout primarily expresses the professionals' experience of being exhausted and unable to give more of themselves (Lacy \& Chan, 2018), it goes beyond this and represents a general response of discontent, dissatisfaction with the work being done frustration with working conditions, and the objectives they are obliged to meet. It includes the experience of many physicians of the conflict between their everyday obligations and their commitment to heal (Wright \& Katz, 2018), which leads to a situation of moral injury (Talbot \& Dean, 2018) or the feeling of having to act in contradiction with their personal convictions.

The prevalence of medical burnout is twice that of other professions, with approximately half the medical population being affected by some of its attendant symptoms (Kumar 2016). The data also indicate that this percentage is increasing (Shanafelt et al., 2019). In the United States, the 2018 Survey of America's Physicians: Practice Patterns and Perspectives has shown that $78 \%$ of physicians have been affected by burnout at some point (Physicians Foundation, 2018). It is hardly surprising then, that it has been regarded as the Hidden Crisis of Health Care (Lacy \& Chan, 2018). According to a report by Harvard University, medical burnout is a "public health crisis that requires urgent action by healthcare institutions, governing bodies, and regulatory authorities." (Jha et al., 2019). From an organizational perspective, in 2016, ten CEOs of the main American health systems declared that medical burnout was a public health crisis (Noseworthy et al., 2017).

From this perspective, physician discontent and the mental problems of the medical professional are not individual problems, but issues caused by a situation, a system, and a task that has become complex and difficult to resolve. Burnout is not a medical condition (Mirkovic \& Bianchi, 2019), but rather a working condition with repercussions on the physical and mental health of medical professionals (World Health Organization, 2019). For this reason, solving the problem should not focus on individual intervention or individual resilience to adversity (Raso, 2020), but rather on changes in the health system and medical practice. The focus of the action should not be primarily to decrease burnout levels but rather to increase the well-being of physicians as a critical factor of personal stability and professional effectiveness (Brower \& Riba, 2017). Satisfaction and enjoyment in the practice of medicine should be the main objective of the health policy to improve physicians' health.

\section{REFERENCES}

Boorse, C. (2016). Goals of medicine. In Giroux, É. (Ed.) Naturalism in the philosophy of health: History, philosophy and theory of the life sciences (pp. 145-177). Switzerland: Spinger.

Brower, K. J., \& Riba, M. B. (2017). Physician mental health and wellbeing: Research and practice. New York: Springer.
Center, C., Davis, M., Detre, T., Ford, D. E., Hansbrough, W., Hendin, H., ... Silverman, M. M. (2003). Confronting depression and suicide in physicians: A consensus statement. JAMA, 289(23), 3161-3166. doi: 10.1001/jama.289.23.3161

Federation of State Medical Boards (FSMB). (2018). Supplemental Resource: Report and Recommendations of the FSMB Workgroup on Physician Wellness and Burnout. Journal of Medical Regulation, 104(2) 37-48. doi: 10.30770/25721852-104.2.37

Firth-Cozens, J. (2003). Doctors, their wellbeing, and their stress. BMJ, 326(7391), 670-671. doi: 10.1136/bmj.326.7391.670

Gazelle, G., Liebschutz, J. M., \& Riess, H. (2015). Physician burnout: Coaching a way out. Journal of General Internal Medicine, 30(4), 508-513. doi: 10.1007/ s11606-014-3144-y

Janus, K., Amelung, V. E., Baker, L. C., Gaitanides, M., Schwartz F. W., \& Rundall T. G. (2008). Job satisfaction and motivation among physicians in academic medical centers: Insights from a cross-national study. Journal of Health Politics, Policy and Law, 33(6), 1133-1167. doi: 10.1215/03616878-2008-035

Jha, A. K., Iliff, A. R., Chaoui, A. A., Defossez, S., Bombaugh, M. C., \& Miller, Y. R. (2019). A crisis in health care: A call to action on physician burnout. Waltham, MA: Massachusetts Medical Society, MHaHA, Harvard TH Chan School of Public Health, and Harvard Global Health Institute.

Kumar, S. (2016). Burnout and Doctors: Prevalence, Prevention and Intervention. Healthcare, 4(3), 37. doi: 10.3390/healthcare4030037

Lacy, B. E., \& Chan, J. L. (2018). Physician burnout: The hidden health care crisis. Clinical Gastroenterology and Hepatology, 16(3), 311-317. doi: 10.1016/j.cgh.2017.06.043

Landrigan, C. P., Barger, L. K., Cade, B. E., Ayas, N. T., \& Czeisler, C. A. (2006). Interns' compliance with accreditation council for graduate medical education work-hour limits. JAMA, 296(9), 1063-1070. doi: 10.1001/jama.296.9.1063

Mache, S., Klapp, B. F., \& Groneberg, D. A. (2014). The well-being and mental health of male and female hospital physicians in Germany. World Hospitals Health Services, 50(4), 17-22. Retrieved from http://www.twmca.org.tw/ upload/site_content_article/30/104071309.pdf\#page $=19$

Maslach, C., \& Jackson, S. E. (1981). The measurement of experienced burnout. Journal of Organizational Behavior, 2(2), 99-113. doi: 10.1002/job.4030020205

Miller, C. (2016). What is the price of physician stress and burnout? Retrieved from: http://www.medicaleconomics.com/medical-economics/news/what-pricephysician-stress-and-burnout

Milner, A., Witt, K., Spittal, M. J., Bismark, M., Graham, M., \& LaMontagne, A. D. (2017). The relationship between working conditions and self-rated health among medical doctors: evidence from seven waves of the Medicine in Australia Balancing Employment and Life (Mabel) survey. BMC Health Services Research, 17(1), 609. doi: 10.1186/s12913-017-2554-Z

Mingote Adán, J. C., Moreno Jiménez, B., Gálvez Herrer., M. (2004). Desgaste profesional y salud de los profesionales médicos: revisión y propuestas de prevención. Medicina Clínica, 123(7), 265-270. doi: 10.1016/S00257753(04)74484-X

Mirkovic, D., \& Bianchi, R. (2019). Physician burnout: let's avoid unsubstantiated claims. Nature Reviews Clinical Oncology, 16(2), 136. doi: 10.1038/s41571018-0150-8

Myers, M., \& Gabbard, G. O. (2008). The Physician as Patient: A Clinical Handbook for Mental Health Professionals. Washington DC: American Psychiatric Publishing.

Noseworthy, J. H. M. J., Madara, J., Cosgrove, D., Edgeworth, M., Ellison, E., Krevans, S., ... Harrison, D. (2017). Physician burnout is a public health crisis: A message to our fellow health care CEOs. Health Affairs Blog, 10. doi: 10.1377/ hblog20170328.059397

Physicians Foundation. (2018). Survey of America's Physicians: Practice Patterns \& Perspectives. Retrieved from https:// physiciansfoundation.org/wp-content/ uploads/ 2018/09/physicians-survey-results-final-2018.pdf

Raso, R. (2020). Burnout: Is resilience enough? Nursing Management, 51(4), 5. doi: 10.1097/01.NUMA.0000657276.13359.65

Schrijver, I., Brady, K. J., \& Trockel, M. (2016). An exploration of key issues and potential solutions that impact physician wellbeing and professional fulfillment at an academic center. PeerJ, 4, e1783. doi: 10.7717/peerj.1783

Shanafelt, T. D., West, C. P., Sinsky, C., Trockel, M., Tutty, M., Satele, D. V., ... Dyrbye, L. N. (2019). Changes in burnout and satisfaction with work-life 
integration in physicians and the general US working population between 2011 and 2017. Mayo Clinic Proceedings, 94(9), 1681-1694. doi: 10.1016/j. mayocp.2018.10.023

Talbot, S. G., \& Dean, W. (2018). Physicians aren't 'burning out.' They're suffering from moral injury. Stat, 7(26), 18. Retrieved from https://www.statnews. com/2018/07/26/physicians-not-burning-out-they -are-suffering-moral-injury/

Tyssen, R. (2018). What is the level of burnout that impairs functioning? Journal of Internal Medicine, 283(6), 594-596. doi: 10.1111/joim.12769

Wallace, J. E. (2012). Mental health and stigma in the medical profession. Health (London), 16(1), 3-18. doi: 10.1177/1363459310371080
Wallace, J. E., Lemaire, J. B., \& Ghali, W. A. (2009). Physician wellness: a missing quality indicator. Lancet, 374(9702), 1714-1721. doi: 10.1016/S01406736(09)61424-0

World Health Organization. (2019). International Statistical Classification of Diseases and Related Health Problems:11th revision (ICD-11). Genève: World Health Organization. Retrieved from https:/www.who.int/classifications/icd/en/

Wright, A. A., \& Katz, I. T. (2018). Beyond burnout—redesigning care to restore meaning and sanity for physicians. $N$ Engl J Med, 378(4), 309-311. doi: 10.1056/NEJMp1716845 